\title{
Optical Spatial Filter to Suppress Beam Wander and Spatial Noise Induced by Atmospheric Turbulence in Free-Space Optical Communications
}

\author{
Ucuk Darusalam, Purnomo Sidi Priambodo, and Eko Tjipto Rahardjo \\ Department of Electrical Engineering, Faculty of Engineering, Universitas Indonesia, Kampus Baru UI, Depok 16424, Indonesia \\ Correspondence should be addressed to Ucuk Darusalam; ucuk.darusalam@gmail.com
}

Received 20 November 2014; Revised 5 April 2015; Accepted 27 April 2015

Academic Editor: Mikhail Noginov

Copyright (C) 2015 Ucuk Darusalam et al. This is an open access article distributed under the Creative Commons Attribution License, which permits unrestricted use, distribution, and reproduction in any medium, provided the original work is properly cited.

\begin{abstract}
We propose an optical spatial filter (OSF) method to suppress beam wander and spatial noise effects. Signal from random displacements of the focus spot around the optical axis within the constricted area is collected. This method advantageously suppresses fluctuations in signal intensity. The OSF consists of a pinhole and cone reflector. The pinhole produces Fresnel diffraction on the focus spot. The cone reflector provides directed reflectance onto the pinhole for random focus spot displacements due to beam wander. The calculations of signal power are based on fluctuations of signal intensity that are minimized by the circular aperture function of the pinhole and the cosine of the reflectance angle from the cone reflector. The method is applied to free-space optical communications at a wavelength of $1.55 \mu \mathrm{m}$ with an atmospheric chamber to provide optical propagation media. Based on calculations, the beam wander angles that can be received by the OSF are from $14.0^{\circ}$ to $28.0^{\circ}$. Moreover, based on experiment, the OSF with a pinhole diameter of $20.0 \mu \mathrm{m}$ and cone reflector diameter of $1.5 \mathrm{~mm}$ produces signal power of $-15.3 \mathrm{dBm}$. Both calculations and experiment show that the OSF enhances the received signal power in the presence of turbulence.
\end{abstract}

\section{Introduction}

Free-space optical (FSO) communications are a class of prospective telecommunication technologies capable of supporting satellite-, terrestrial-, high altitude-, and mobile terminal-platforms that have been developed worldwide, recently [1-4]. However, atmospheric turbulence can cause severe problems that degrade FSO performance, such as decreased signal-to-noise ratio (SNR) and increased biterror-rate (BER) $[5,6]$. Turbulence causes optical propagation phenomena such as diffraction, scattering, absorption, and beam spreading that attenuate or diffuse the FSO signal through stochastic processes. The FSO experiences spatial noise or random movement of hot spots around the center of the focus spot in the receiver plane [7]. Turbulence also produces beam wander that fluctuates the angle-of-arrival on the receiver lens, leading to random movements of the focus spot [8]. Beam wander and spatial noise are turbulence effects on optical propagation that become major factors in fluctuating signal intensity.
Several methods have been developed to overcome turbulence effects. These are spatial-diversity [9], time-diversity [10], cooperative diversity [11], optical amplification [12], photo sensor design [13], and adaptive optics [14]. The aforementioned methods have several common drawbacks, such as a high complexity and decreased bit-rate-capacity processing. For example, spatial-diversity involves transmission or reception of multiple beams to produce high signal intensity with minimum noise and requires complex electronics to achieve equal gain combining. Time-diversity uses a complex of decoding techniques to receive redundant signals over a period of time. Thus, spatial- and time-diversity use complex signal processing that risk decreasing the bit-rate-capacity if signal intensity fluctuations are not minimized beforehand. However, since these methods use direct- or fiber-detection to retrieve signals, optics suppressing beam wander and spatial noise may be implemented before the receiver plane [15] to minimize signal intensity fluctuations.

Beam wander and spatial noise cannot be treated as separate and independent processes, since they both occur 
in the focus spot $[16$, p. 201]. They increase with propagation path length and turbulence level to cause signal intensity fluctuations. Thus, under atmospheric turbulence, signal power often drops below the prescribed threshold level of receiving photodetectors (PD). In order to address fluctuation of signal intensity, beam wander and spatial noise must be treated as an integrated process. An optical method of correcting for beam wander and spatial noise may be implemented at the focus spot before signal detection by PD. Optical signal pre-processing methods have the advantages of fast response, low-cost, and simplicity over other methods. They do not limit bit-rate-capacity processing. They can also be implemented to optimize amplification method that commonly uses erbium-doped fiber amplifier (EDFA).

We propose an OSF as the optical method to suppress beam wander and spatial noise effects before the signal is received by PD. In [15], an optical method based on a Fourier optics filter pinhole was developed to suppress spatial noise. However, this method did not address beam wander and spatial noise simultaneously. For example, it could not solve the misalignment of the incoming signal caused by beam wander. Considering this limitation, the OSF proposed here is designed to suppress beam wander and spatial noise effects simultaneously. This suppression is achieved by acquiring signal within a small area, while random displacement of the focus spot may be collected in this region. This concept of the OSF has the advantage of suppressing fluctuation of signal intensity. Hence, PD receives minimal signal intensity fluctuation, amplifying signal power rather than noise. The OSF is composed of a cone reflector and pinhole and implemented at the focus spot. Furthermore, calculations of signal power based on the mean signal intensity from the OSF under the influence of turbulence effects are carried out. In order to confirm our calculations, an experiment of the OSF implemented in an FSO system is also carried out, where the optical signal propagates through turbulent media. For analysis, the signal power resulting from the OSF is compared to that of a direct-detection (DD) method through calculations and experiment.

\section{The Optical Spatial Filter: Pinhole and Cone Reflector}

The configuration of the OSF optical system is shown in Figure 1. It is composed of four planes: a transmitter plane $\mathbf{X}_{-2}$, a receiver lens plane $\mathbf{X}_{-1}$, the OSF plane $\mathbf{X}_{0}$, and a receiver plane $\mathbf{X}_{1}$. The origin $z_{0}=0$ is located at $\mathbf{X}_{0}$. Optical propagation from $\mathbf{X}_{-2}$ to $\mathbf{X}_{-1}$ in the $+z$ direction is subject to atmospheric turbulence. Fluctuation of signal intensity at $\mathbf{X}_{-1}$ is mainly caused by beam wander and spatial noise effects. Beam wander is the fluctuation of the incident beam angle-of-arrival at the receiver lens $\mathbf{X}_{-1}$ that leads to random displacement of the focus spot around the optical axis. Spatial noise is the fluctuation of phase distribution that occurs during optical propagation leading to random movement of hot spots around the center of the focus spot. Incident light is focused by the receiver lens at $\mathbf{X}_{-1}$ onto the pinhole of the OSF. The mean irradiance of focus spot coincident with

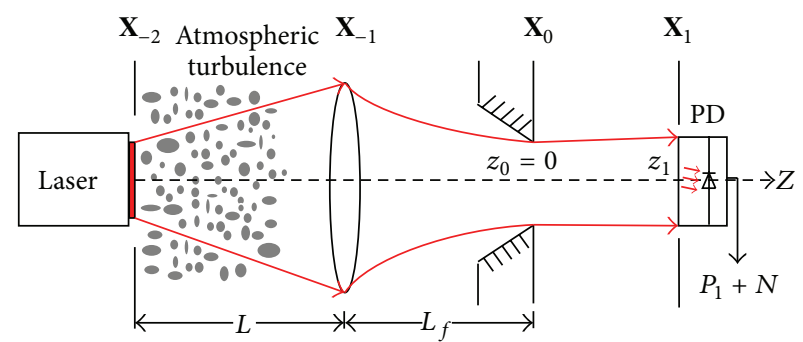

FIGURE 1: Optical system configuration of the OSF for suppression of beam wander and spatial noise effects induced by atmospheric turbulence.

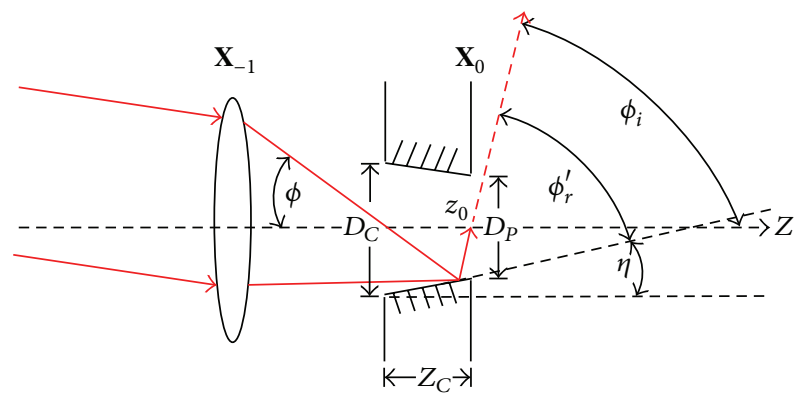

FIGURE 2: Cone reflector of the OSF for suppression angle of beam wander. Incoming angle $\phi$ produces incident angle $\phi_{i}$ at the output of the pinhole.

the pinhole following atmospheric turbulence [16, page 459] is given below:

$$
\left\langle I_{0}\left(\mathbf{r}_{0}, 0\right)\right\rangle=\frac{W_{G}^{2}}{W_{0}^{2}} \operatorname{SR} I_{-1}^{0}\left(0,-L_{f}\right) \exp \left(-\operatorname{SR} \frac{2 r_{0}^{2}}{W_{0}^{2}}\right),
$$

where $\langle\cdot\rangle$ denotes mean value, $\mathbf{r}_{\mathbf{0}}$ is the radial coordinate of the pinhole on $\mathbf{X}_{\mathbf{0}}, I_{-1}^{0}\left(0,-L_{f}\right)$ is the free-space irradiance, $W_{G}$ is the effective aperture radius of receiver lens, $W_{0}$ is the focus spot radius, and SR is the Strehl ratio. The pinhole radius is $0 \leq r_{0} \leq D_{P} / 2$, where $D_{P}$ is the pinhole diameter, as shown in Figure 2. The Strehl ratio SR is formulated as $1 /(1+$ $\left.1.63 \sigma_{R}^{12 / 5}(L) \Lambda_{-1}\right)$, where term $\sigma_{R}^{12 / 5}(L)$ is the Rytov variance for propagation path length $L$ and $\Lambda_{-1}$ is the effective beam parameter. Based on [16], $\sigma_{R}^{12 / 5}(L)=\left(1.23 C_{n}^{2} k^{7 / 6} L^{11 / 6}\right)^{6 / 5}$ and $\Lambda_{-1}=2 L / k W_{-1}^{2}$, where $W_{-1}$ is the incident light radius on the receiver lens and $k=2 \pi / \lambda$ is the wave number. In (1), $\left\langle I_{0}\left(\mathbf{r}_{0}, 0\right)\right\rangle$ is the mean signal intensity, modulated by beam wander and spatial noise effects.

Beam wander and spatial noise effects degrade signal intensity $\left\langle I_{1}\right\rangle$. Thus, PD produces less signal power $P_{1}$ and amplifies noise. In order to suppress spatial noise, the OSF includes a pinhole at $\mathbf{X}_{0}$ to control diffraction. It produces 
a near-field Fresnel diffraction distribution on $\mathbf{X}_{1}$ at $z_{1}$. The mean signal intensity at $z_{1}[17]$ is

$$
\begin{aligned}
\left\langle I_{1}\left(\mathbf{r}_{1}, z_{1} \approx 0\right)\right\rangle & \\
= & \frac{1}{4} \frac{W_{G}^{2}}{W_{0}^{2}} \operatorname{SR} I_{-1}^{0}\left(0,-L_{f}\right) \exp \left(-\operatorname{SR} \frac{2 r_{0}^{2}}{W_{0}^{2}}\right) \\
\quad & \times\left[1-2 \cos (v) J_{0}(v)+J_{0}^{2}(v)\right],
\end{aligned}
$$

where $J_{0}$ is a Bessel function of the first kind and $v=$ $\left[2 \pi\left(D_{P} / 2\right) / \lambda z_{1}\right] r_{1}$. In (2), spatial noise on the focus spot is suppressed by the last term $\left[1-2 \cos (v) J_{0}(v)+J_{0}^{2}(v)\right]$.

A cone reflector reflects incoming beams wandering with angle $\phi$, as shown in Figure 2. It has a radial mirror with diameter $D_{C}$, width $Z_{C}$, and tilt angle $\eta$. Incoming angle $\phi$, under atmospheric turbulence, is random. The radial mirror reflects an incoming focus spot deflected at $\phi$, into complementary angle $\phi_{r}^{\prime}$. This produces an incident angle $\phi_{i}$ with respect to optical axis $Z$ at the output of the pinhole at $\mathbf{X}_{0} \cdot \phi_{i}$ is given by

$$
\phi_{i}=\phi_{r}^{\prime}+\eta=\phi+2 \eta=\phi+2 \tan ^{-1}\left(\frac{D_{C}-D_{P}}{2 Z_{C}}\right),
$$

where $\eta$ is tilt angle of the cone reflector with respect to the optical axis $Z$. $\phi$ in (3) ranges from $\phi_{\min }$ to $\phi_{\max }$ given by

$$
\begin{aligned}
& \phi_{\min }=\phi^{0}+\tan ^{-1}\left(\frac{(1 / 2)\left(D_{G}-D_{P}\right)}{L_{f}}\right), \\
& \phi_{\max }=\phi_{\min }+\tan ^{-1}\left(\frac{(1 / 2)\left(D_{G}-D_{C}\right)}{L_{f}-Z_{C}}\right),
\end{aligned}
$$

where $\phi^{0}$ is the maximum angle of the focus spot unaffected by turbulent atmosphere, $D_{G}$ is the hard diameter of the receiver lens, and $L_{f}$ is the length of the focus spot. Thus, the cone reflector reflects randomly displaced focus spot to be reflected onto pinhole diameter. Accounting for angle of incidence $\phi_{i}$ in (3), $I_{1}\left(\mathbf{r}_{1}, z_{1} \approx 0\right)$ in (2) should be multiplied by $\cos \left(\phi_{i}\right)$ :

$$
\begin{aligned}
&\left\langle I_{1}\left(\mathbf{r}_{1}, z_{1} \approx 0, \phi_{i}\right)\right\rangle \\
&= \frac{1}{4} \frac{W_{G}^{2}}{W_{0}^{2}} \operatorname{SR} I_{-1}^{0}\left(0,-L_{f}\right) \exp \left(-\operatorname{SR} \frac{2 r_{0}^{2}}{W_{0}^{2}}\right) \cos \left(\phi_{i}\right) \\
& \quad \times\left[1-2 \cos (\nu) J_{0}(v)+J_{0}^{2}(v)\right] .
\end{aligned}
$$

Assuming that $\mathbf{X}_{0}$ and $\mathbf{X}_{1}$ are almost coincident, such that $z_{0} \approx z_{1}$ and $\mathbf{r}_{0} \approx \mathbf{r}_{1}$, the $\mathrm{PD}$ receives instantaneous signal intensity $I_{1}\left(\mathbf{r}_{1}, z_{1} \approx 0, \phi_{i}\right)$ from the pinhole. The $\mathrm{PD}$ receives the fundamental component of the diffracted intensity to produce optimum signal power $P_{1}$. The mean signal power $\left\langle P_{1}\right\rangle$ is

$$
\begin{aligned}
& \left\langle P_{1}\left(\mathbf{r}_{1}, z_{1} \approx 0, \phi_{i}\right)\right\rangle \\
& \quad=\frac{\pi}{2} \frac{W_{G}^{2}}{W_{0}^{2}} \operatorname{SR~} I_{-1}^{0}\left(0,-L_{f}\right) \exp \left(-\operatorname{SR} \frac{2 r_{0}^{2}}{W_{0}^{2}}\right) \cos \left(\phi_{i}\right) B,
\end{aligned}
$$

where $B$ is the circular aperture function of pinhole

$$
\begin{aligned}
B & =\frac{r_{1}^{2}}{2}-\left[\frac{2 A^{2} r_{1}^{2}}{3} \cos \left(A r_{1}\right) J_{0}\left(A r_{1}\right)\right. \\
& \left.+\frac{A^{2} r_{1}^{2} \sin \left(A r_{1}\right)-A r_{1} \cos \left(A r_{1}\right)}{3} J_{1}\left(A r_{1}\right)\right] \\
& +\left[\frac{A^{2} r_{1}^{2}}{2}\left(J_{0}^{2}\left(A r_{1}\right)+J_{1}^{2}\left(A r_{1}\right)\right)\right] .
\end{aligned}
$$

$A=\left[2 \pi\left(D_{P} / 2\right) / \lambda z_{1}\right]$ is the spatial frequency of the focus spot within the pinhole diameter and $J_{1}$ is the Bessel function of the second kind.

In comparison to the DD method, which, as stated in [16, p. 459], suffers from degrading signal power with atmospheric turbulence, $\left\langle P_{1}\right\rangle$ in (6) has minimal fluctuation of signal intensity. This is due to $B$ and $\cos \left(\phi_{i}\right)$ in (5). $B$ modulates and limits spatial noise on the area of the pinhole. Thus, spatial noise is confined to a limited area. $\cos \left(\phi_{i}\right)$ compensates for fluctuations of signal intensity caused by random displacement of the focus spot. Due to these operations, signal intensity $\left\langle I_{1}\right\rangle$ received by the $\mathrm{PD}$ increases. The pinhole operates as a low-pass filter to spatial noise, while the cone reflector compensates for signal intensity that would otherwise be lost to beam wander. The reflection from cone reflector guides randomly displacement light to the pinhole. Through this, the OSF suppresses beam wander and spatial noise simultaneously to minimize fluctuations in signal intensity.

\section{Experimental Setup}

In this experiment, FSO with full-duplex transmission operates at wavelength $\lambda=1.55 \mu \mathrm{m}$, as shown in Figure 3, at a 1 Gbps bit-rate with on-off keying modulation. A box of turbulence simulator (BTS) acts as an atmospheric chamber, as shown in Figure $4[18,19]$, that provides turbulent media for optical propagation in the FSO. The BTS has dimensions of $4.0 \mathrm{~m} \times 0.5 \mathrm{~m} \times 0.5 \mathrm{~m}$ and operates by mixing flows of a highto-cold temperature-gradient. Steam at $T_{1}=100^{\circ} \mathrm{C}$ and lowspeed and cold air at $T_{2}=16^{\circ} \mathrm{C}$ and $6.0-8.0 \mathrm{~m} / \mathrm{s}$ are allowed to combine inside; the flows are broken by ailerons inside of the BTS. These processes produce turbulent media that affects the optical propagation of the forward-directed signal within the BTS. In order to avoid interference in the optical propagation, backward-directed signal that is conditioned in non-turbulent media is separated from forward-directed signal.

In the experiment, $W_{-2}=0.015 \mathrm{~m}$ is the transmitted light radius from laser source, $W_{-1} \cong 0.15 \mathrm{~m}$, and $W_{0}=500 \mu \mathrm{m}$. The signal is transmitted with power $P_{-2}^{0}$ of $+16.5 \mathrm{dBm}$ from beam collimator BC-2 to receiver lens-2, as shown in Figure 3. The receiver lens has $D_{G}=0.05 \mathrm{~m}$ and $L_{f}=0.1 \mathrm{~m} . P_{\mathrm{Th}}$ of $-25.0 \mathrm{dBm}$ is the threshold level of the PD. The optical power meter (OPM) is used to measure signal power $P_{1}$.

The OSF has $D_{C}=1.5 \mathrm{~mm}$ and $Z_{C}=2.0 \mathrm{~mm}$. It is designed with various pinhole diameters of $50.0 \mu \mathrm{m}, 40 \mu \mathrm{m}$, $30 \mu \mathrm{m}$, and $20 \mu \mathrm{m}$ for $D_{P 1}, D_{P 2}, D_{P 3}$, and $D_{P 4}$, respectively. 


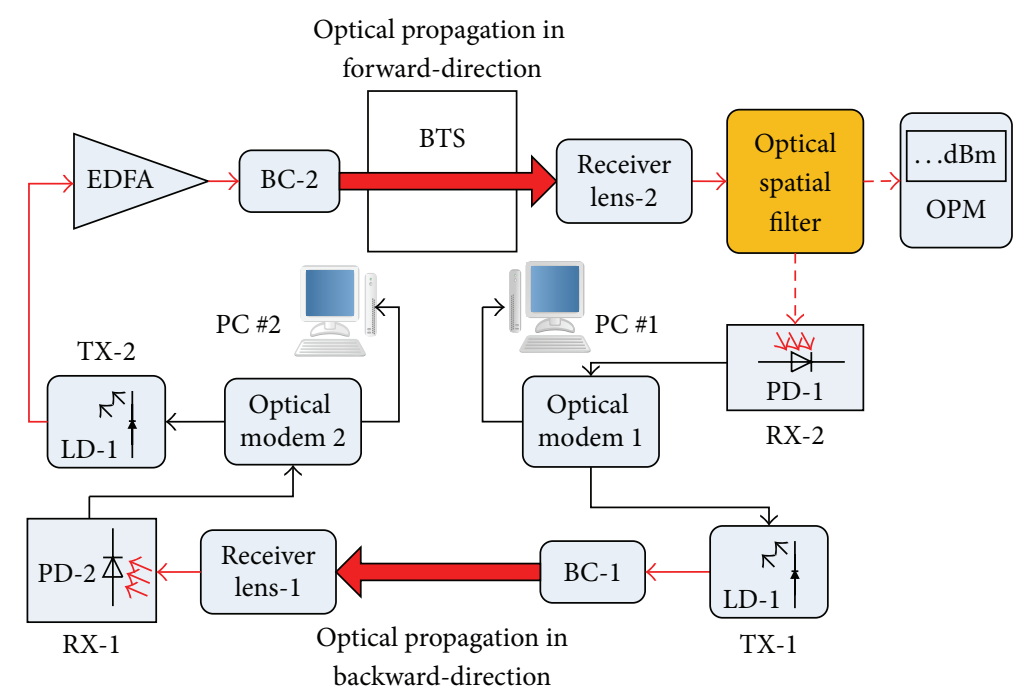

FIGURE 3: Full-duplex transmitting FSO operating at a wavelength of $1.55 \mu \mathrm{m}$ and a data rate of 1 Gbps of data rate with turbulent media produced by the BTS.

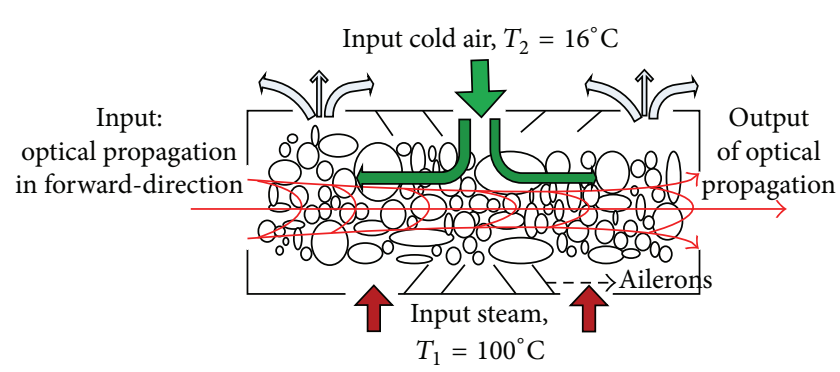

FIGURE 4: Box of turbulence simulator (BTS) providing turbulent media to affect forward-directed optical propagation.

Based on (3), tilt angles $\eta$ are $19.9^{\circ}, 20.0^{\circ}, 20.2^{\circ}$, and $20.3^{\circ}$ for $D_{P 1}, D_{P 2}, D_{P 3}$, and $D_{P 4}$, respectively. Since the different values of $\eta$ are low for all $D_{P}$, the range of beam wander angles that can be received by the cone reflector is $14.0^{\circ}$ to $38.0^{\circ}$, according to (4). The cone reflector guides random angles $\phi$ within that range onto the pinhole. The OSF is made of silver in order to produce optimum reflection at $1.55 \mu \mathrm{m}$ [20]. The OSF is installed at $z_{0}=0$ on $\mathbf{X}_{0}$. The PD is installed at $\mathbf{X}_{1}$ at the output of the OSF, where $z_{1} \approx 0$. Hence, the PD receives the fundamental component of diffracted light from the pinhole of the OSF.

\section{Results and Discussion}

Calculated signal power $\left\langle P_{1}\right\rangle$ for the OSF and DD methods is shown in Figure 5. Based on the experiment, signal power from the laser source at $\mathbf{X}_{-2}$ in the absence of turbulence media of the BTS, $P_{-2}^{0}=+16.5 \mathrm{dBm}$, is chosen as a reference for the analysis. The calculations are based on the value of the index structure of strong turbulence, $C_{n}^{2}=5 \times 10^{-13} \mathrm{~m}^{-2 / 3}$. Considering (4) and the parameters of the OSF, beam wander angles of $18^{\circ}, 28^{\circ}$, and $38^{\circ}$ are chosen for $\phi_{1}, \phi_{2}$, and

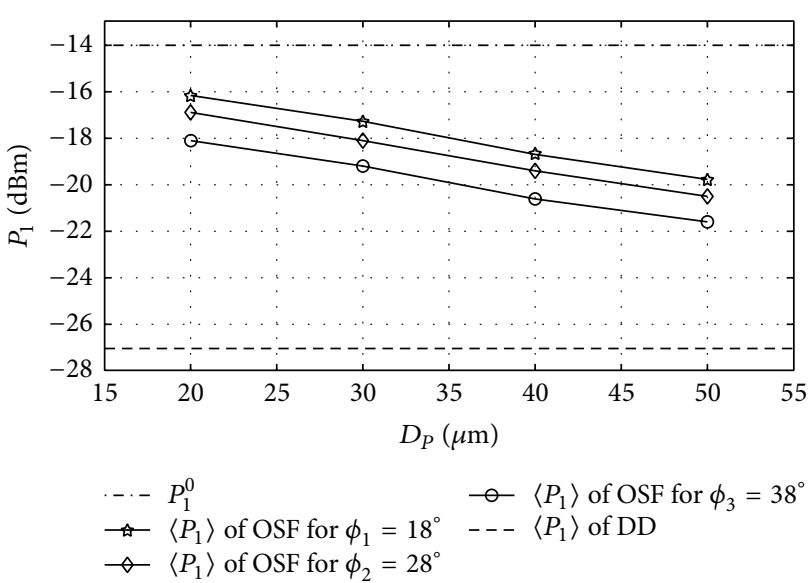

Figure 5: OSF signal power $\left\langle P_{1}\right\rangle$ versus pinhole diameter $D_{P}$ for beam wander angles $\phi_{1}=18.0^{\circ}, \phi_{2}=28.0^{\circ}$, and $\phi_{3}=38.0^{\circ}$, where $\left\langle P_{1}\right\rangle=-27.0 \mathrm{dBm}$ is the power in the DD method and $P_{1}^{0}=-14.0 \mathrm{dBm}$ is the signal power in the absence of atmospheric turbulence.

$\phi_{3}$, respectively, in the calculations. The properties of the optical propagation and optical system are set based on the experiment.

As shown in Figure 5, signal power $\left\langle P_{1}\right\rangle$ degrades significantly in the DD method, falling below $P_{\mathrm{Th}}$. Relative to $P_{1}^{0}=-14 \mathrm{dBm}$, signal power $\left\langle P_{1}\right\rangle$ in the DD method is not too low to be detected by the PD after the signal intensity $I_{0}$ is modulated by beam wander and spatial noise. However, for the OSF, signal power $\left\langle P_{1}\right\rangle$ exceeds $P_{\mathrm{Th}}$. Figure 5 shows that signal power $\left\langle P_{1}\right\rangle$ of the OSF is greater for all $D_{P}$ than that of the DD method. It also shows that signal power $\left\langle P_{1}\right\rangle$ is higher for the OSF in the case of beam wander angles $\phi_{1}, \phi_{2}$, and $\phi_{3}$. Considering values of $\phi^{0}, \phi_{\min }$, and $\phi_{\max }$ in the experiment, it can be known that $\phi$ of $28.0^{\circ}$ to $38.0^{\circ}$ is the range of beam 


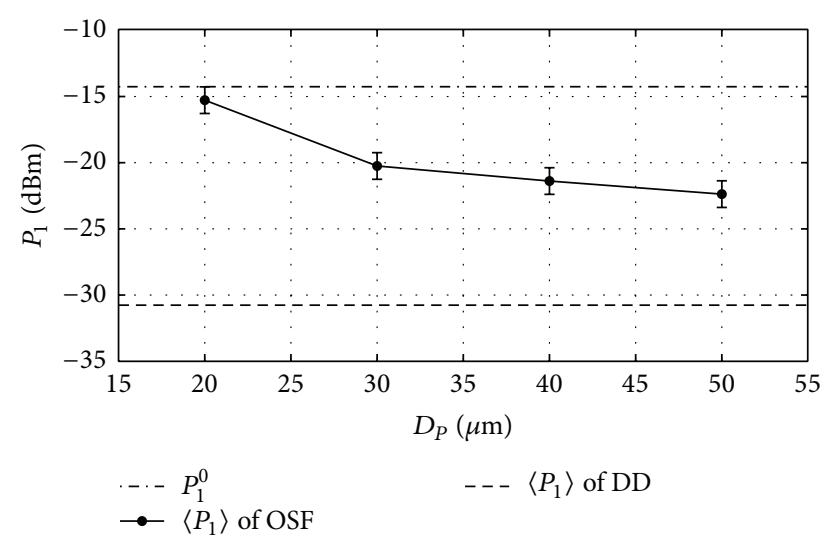

FIGURE 6: Experimentally signal power $\left\langle P_{1}\right\rangle$ versus pinhole diameter $D_{P}$ for the DD and the OSF methods, where $\left\langle P_{1}\right\rangle=-31.0 \mathrm{dBm}$ is from $\mathrm{DD}$ method and $P_{1}^{0}=-14.0 \mathrm{dBm}$ is signal power in the absence of atmospheric turbulence.

wander angles that can be received by the cone reflector and be reflected onto the pinhole. Beam wander angles $\phi$ of $14.0^{\circ}$ to $27.0^{\circ}$ directly reach the pinhole without reflection off the cone reflector. Thus, the OSF receives beam wander angles in the range of $14.0^{\circ}$ to $38.0^{\circ}$. The term of $\cos \left(\phi_{i}\right)$ in (5) compensates for signal intensity $\left\langle I_{1}\right\rangle$ that would otherwise be lost to beam wander. Signal power $\left\langle P_{1}\right\rangle$ is higher for the OSF at $\phi_{2}$ and $\phi_{3}$ than for the DD method. Moreover, signal power is produced highest by the OSF at $\phi_{1}$. Figure 5 shows that the OSF suppresses fluctuations of signal intensity as $D_{P}$ gets smaller. Based on (5), the circular aperture function of pinhole $B$ filters spatial noise through localization to a small area. Thus, the circular aperture function of $B$ works best for smaller pinhole diameters, as seen in (7).

As shown in Figure 6, the DD method produces lower signal power in the presence of turbulent media, $\left\langle P_{1}\right\rangle=$ $-31.0 \mathrm{dBm}$, well below $P_{\mathrm{Th}}$. Turbulent media of the BTS induces beam wander and spatial noise in the forwarddirected optical propagation. This random process leads to fluctuation of signal intensity $I_{0}$. Hence, average signal intensity $\left\langle I_{0}\right\rangle$ decreases. Moreover, this condition leads to performance degradation such that the PD minimizes signal power and maximizes noise. The OSF suppresses beam wander and spatial noise effects, as shown in Figure 6. Signal power $\left\langle P_{1}\right\rangle$ is well above $P_{\mathrm{Th}}$. Moreover, $\left\langle P_{1}\right\rangle$ for the OSF is higher for all $D_{P}$ than it is for the DD method, even approaching $P_{1}^{0} .\left\langle P_{1}\right\rangle$ from the OSF is $-22.4 \mathrm{dBm}$, $-21.4 \mathrm{dBm},-20.3 \mathrm{dBm}$, and $-15.3 \mathrm{dBm}$ for $D_{P 1}, D_{P 2}, D_{P 3}$, and $D_{P 4}$, respectively. Beam wander and spatial noise effects are effectively suppressed by the cone reflector and pinhole to enhance $\left\langle I_{1}\right\rangle$, as stated in (5). Spatial noise created by optical propagation through turbulence is filtered out by the pinhole. Therefore within the limited area of the pinhole, spatial noise is limited. Meanwhile, random displacement of the focus spot is minimized by the cone reflector. Thus, offaxis light is collected within the pinhole, as well. Between the pinhole and cone reflector signal intensity fluctuation is optimally suppressed. Turbulence effects are stochastic and independent processes that cause random beam wander.
The cone reflector suppresses beam wander, minimizing the effects of misalignment of light collected by the PD.

By experiment, as shown in Figure 6, different values of $D_{P}$ tend to produce the same value of signal power $\left\langle P_{1}\right\rangle$. This is particularly true for $D_{P 1}, D_{P 2}$, and $D_{P 3}$. In the experiment, the BTS produces turbulence with a scale or level that has random variations. We assume that, for $D_{P 4}$, the turbulence level is not different than for the other pinhole diameters, so $D_{P 4}$ produces the highest signal power $\left\langle P_{1}\right\rangle$. Thus, calculation and experiment show a similar trend of smaller pinholes better suppressing of signal intensity fluctuations. Moreover, the OSF, for all pinhole diameters, minimizes signal intensity fluctuations $\left\langle I_{1}\right\rangle$ and maximizes signal power $\left\langle P_{1}\right\rangle$ relative to the DD method.

Based on the results of calculations and experiment, as shown in Figures 5 and 6, the OSF enhances signal intensity $\left\langle I_{1}\right\rangle$ that was degraded significantly by turbulence effects in the DD method. Beam wander and spatial noise effects are suppressed by the cone reflector and pinhole. Through this suppression, greater signal intensity $I_{1}$ is received by the PD. By (5), fluctuation of signal intensity caused by beam wander and spatial noise effects can be minimized, as well. Relative to signal power in the absence of turbulent media in the experiment, $P_{1}^{0}=-14.0 \mathrm{dBm}$, the OSF improves over the DD method, producing higher signal power $\left\langle P_{1}\right\rangle$. Thus, the OSF better suppresses fluctuation of signal intensity $\left\langle I_{0}\right\rangle$, relative to the DD method.

According to the experiment, the OSF produces greater signal power $\left\langle P_{1}\right\rangle$ than the DD method by $8.6 \mathrm{~dB}, 9.6 \mathrm{~dB}$, $10.7 \mathrm{~dB}$, and $15.7 \mathrm{~dB}$ for $D_{P 1}, D_{P 2}, D_{P 3}$, and $D_{P 4}$, respectively. The OSF enhances signal intensity $\left\langle I_{1}\right\rangle$ to produce higher signal power $\left\langle P_{1}\right\rangle$ by suppressing beam wander and spatial noise effects induced by optical propagation through turbulence media. It keeps signal power $\left\langle P_{1}\right\rangle$ above $P_{\text {Th }}$, ensuring signal detection over noise. The OSF localizes the acquisition of focus spot to the small area of the pinhole in order to filter spatial noise. Thus, fluctuation of signal intensity caused by spatial noise is limited to the small area of pinhole, as well. The pinhole causes Fresnel diffraction, as in the circular aperture function, $B$, of (5). The pinhole filters spatial noise but can also decrease signal intensity $\left\langle I_{1}\right\rangle$, as shown in (2). The cone reflector compensates for signal intensity loss by collecting displaced focus spot onto the pinhole. Thus, fluctuation of signal intensity is minimized within the pinhole diameter. In order to optimize pinhole operation for suppression of spatial noise, the ratio of pinhole diameter $D_{P}$ and the space between the OSF and the PD, $z_{1}$, should satisfy $\left(D_{P} / 2\right)=\lambda z_{1} / 4 r_{1}$.

The tilt angle of the cone reflector, $\eta$, plays an important role in reflecting randomly displaced focus onto the pinhole. Based on (3), dimensions of cone reflector must be such that $2 \tan ^{-1}\left(D_{C}-D_{P} / 2 Z_{C}\right)$ minimizes $\phi_{i}$. Hence, the maximum possible $\eta$ is limited by the requirement that $\tan ^{-1}\left(D_{C}-\right.$ $\left.D_{P} / 2 Z_{C}\right)<\phi$, since $\phi$ cannot be $\pi / 2$ in FSO. Based on (4), the range of $\phi_{\min }$ to $\phi_{\max }$ can be maximized by choosing optimum parameters of $D_{C}, D_{P}, Z_{C}$, and $L_{f}$, allowing for greater $\phi$. Thus, larger $\phi$ for strong turbulence levels can be collected onto the pinhole, further enhancing signal intensity. Thus, random displacement of the focus spot from the PD that 
limits the DD method under turbulence can be minimized by our method.

The OSF is a competitive method to be implemented in optical amplification. For example, for erbium-doped fiber amplifiers (EDFA) used in FSO, noise can decrease SNR and increase BER [21]. Since the focus spot is directly input into the EDFA in the forward-relaying scheme, beam wander and spatial noise effects are amplified with the signal. Thus, the signal gain by EDFA is not optimized over noise. SNR by EDFA degrades, since maximum noise is also amplified, even in the saturated region of the amplification process. Thus, the OSF can be implemented before the EDFA to suppress beam wander and spatial noise effects before amplification. Integrating OSF and EDFA should minimize signal intensity fluctuations and maximize signal. Furthermore, higher signal power can be produced by EDFA with minimal noise. By this scheme, the signal intensity will be maximized over noise. Hence, SNR should increase and BER should decrease.

\section{Conclusions}

The suppression of beam wander and spatial noise effects by the OSF has been demonstrated by calculation and experiment. The OSF produces higher signal power in the presence of turbulence than the DD method does. Suppression of beam wander and spatial noise effects is optimized by smaller pinhole diameters for the OSF. The OSF suppresses beam wander and spatial noise effects simultaneously, greatly decreasing signal intensity fluctuations over the DD method. Thus, the OSF produces higher average signal intensity $\left\langle I_{1}\right\rangle$ than DD method.

\section{Conflict of Interests}

The authors declare no conflict of interests regarding the publication of this paper.

\section{References}

[1] V. W. S. Chan, "Free-space optical communications," Journal of Lightwave Technology, vol. 24, no. 12, pp. 4750-4762, 2006.

[2] K. Wakamori, K. Kazaura, and I. Oka, "Experiment on regional broadband network using free-space-optical communication systems," Journal of Lightwave Technology, vol. 25, no. 11, pp. 3265-3273, 2007.

[3] W. J. Miniscalco and S. A. Lane, "Optical space-time division multiple access," Journal of Lightwave Technology, vol. 30, no. 11, pp. 1771-1785, 2012.

[4] R. Paudel, Z. Ghassemlooy, H. Le-Minh, and S. Rajbhandari, "Modelling of free space optical link for ground-to-train communications using a Gaussian source," IET Optoelectronics, vol. 7, no. 1, pp. 1-8, 2013.

[5] J. Park, E. Lee, and G. Yoon, "Average bit-error rate of the alamouti scheme in gamma-gamma fading channels," IEEE Photonics Technology Letters, vol. 23, no. 4, pp. 269-271, 2011.

[6] M. Niu, J. Cheng, and J. F. Holzman, "Error rate analysis of Mary coherent free-space optical communication systems with $\mathrm{K}$ distributed turbulence," IEEE Transactions on Communications, vol. 59, no. 3, pp. 664-668, 2011.
[7] J. Recolons, L. C. Andrews, and R. L. Phillips, "Analysis of beam wander effects for a horizontal-path propagating Gaussianbeam wave: focused beam case," Optical Engineering, vol. 46, no. 8, Article ID 086002, pp. 1-11, 2007.

[8] Y. Ren, A. Dang, B. Luo, and H. Guo, "Capacities for longdistance free-space optical links under beam wander effects," IEEE Photonics Technology Letters, vol. 22, no. 14, pp. 1069-1071, 2010.

[9] M.-A. Khalighi, N. Schwartz, N. Aitamer, and S. Bourennane, "Fading reduction by aperture averaging and spatial diversity in optical wireless systems," Journal of Optical Communications and Networking, vol. 1, no. 6, pp. 580-593, 2009.

[10] F. Xu, A. Khalighi, P. Caussé, and S. Bourennane, "Channel coding and time-diversity for optical wireless links," Optics Express, vol. 17, no. 2, pp. 872-887, 2009.

[11] C. Abou-Rjeily and A. Slim, "Cooperative diversity for freespace optical communications: transceiver design and performance analysis," IEEE Transactions on Communications, vol. 59, no. 3, pp. 658-663, 2011.

[12] M. A. Kashani, M. M. Rad, M. Safari, and M. Uysal, "Alloptical amplify-and-forward relaying system for atmospheric channels," IEEE Communications Letters, vol. 16, no. 10, pp. 1684-1687, 2012.

[13] C. Rivera and M. Álvarez, "Assessment of PbSe photoconductors for the realization of free-space midinfrared optical communication links," IEEE Photonics Technology Letters, vol. 24, no. 4, pp. 267-269, 2012.

[14] Y. Yuan, Y. Cai, J. Qu, H. T. Eyyuboǧlu, and Y. Baykal, "Average intensity and spreading of an elegant Hermite-Gaussian beam in turbulent atmosphere," Optics Express, vol. 17, no. 13, pp. 11130-11139, 2009.

[15] P. S. Priambodo, U. Darusalam, and E. T. Rahardjo, "Free-space optical propagation noise suppression by Fourier optics filter pinhole," International Journal of Optics and Applications, vol. 5, pp. 27-32, 2015.

[16] L. C. Andrews and R. L. Phillips, Laser Beam Propagation through Random Media, SPIE Press, Philadelphia, Pa, USA, 2nd edition, 2005.

[17] H.-E. Hwang, G.-H. Yang, and P. Han, "Study and improvement of near-field diffraction limits of circular aperture imaging systems," Journal of the Chinese Institute of Engineers, vol. 25, no. 3, pp. 335-340, 2002.

[18] J. Perez, Z. Ghassemlooy, S. Rajbhandari, M. Ijaz, and H. Le Minh, "Ethernet FSO communications link performance study under a controlled fog environment," IEEE Communications Letters, vol. 16, no. 3, pp. 408-410, 2012.

[19] M. Ijaz, Z. Ghassemlooy, J. Perez, V. Brazda, and O. Fiser, "Enhancing the atmospheric visibility and fog attenuation using a controlled FSO channel," IEEE Photonics Technology Letters, vol. 25, no. 13, pp. 1262-1265, 2013.

[20] J. M. Bennett and E. J. Ashley, "Infrared reflectance and emittance of silver and gold evaporated in ultrahigh vacuum," Applied Optics, vol. 4, no. 2, pp. 221-224, 1965.

[21] Y.-S. Hurh, K.-W. Shin, S.-H. Lee, and J.-S. Lee, "Weatherinsensitive optical free-space communication using gainsaturated optical fiber amplifiers," Journal of Lightwave Technology, vol. 23, no. 12, pp. 4022-4025, 2005. 

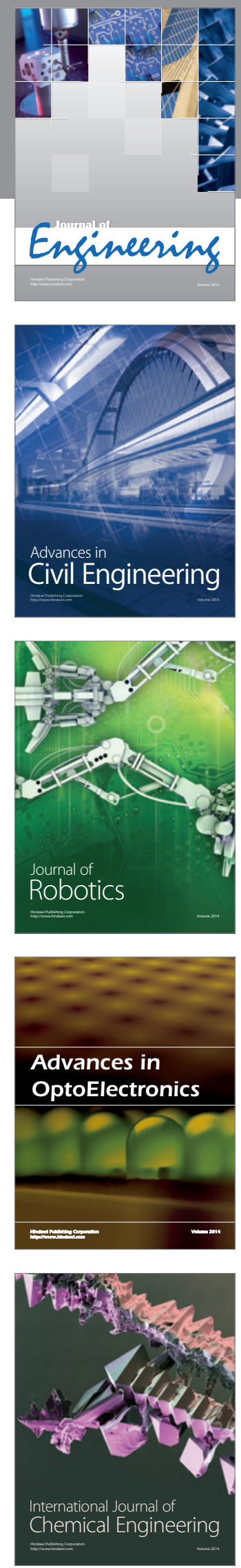

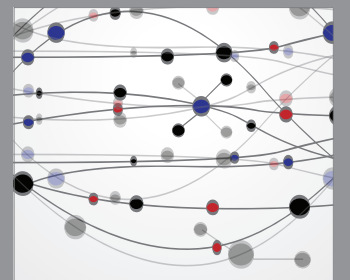

The Scientific World Journal
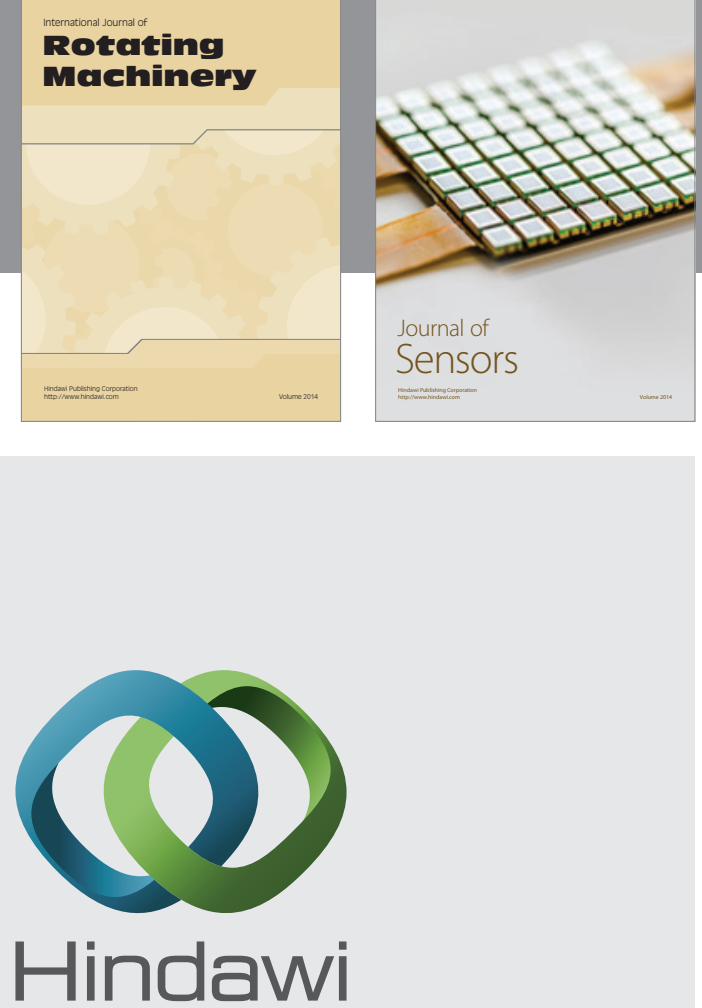

Submit your manuscripts at http://www.hindawi.com
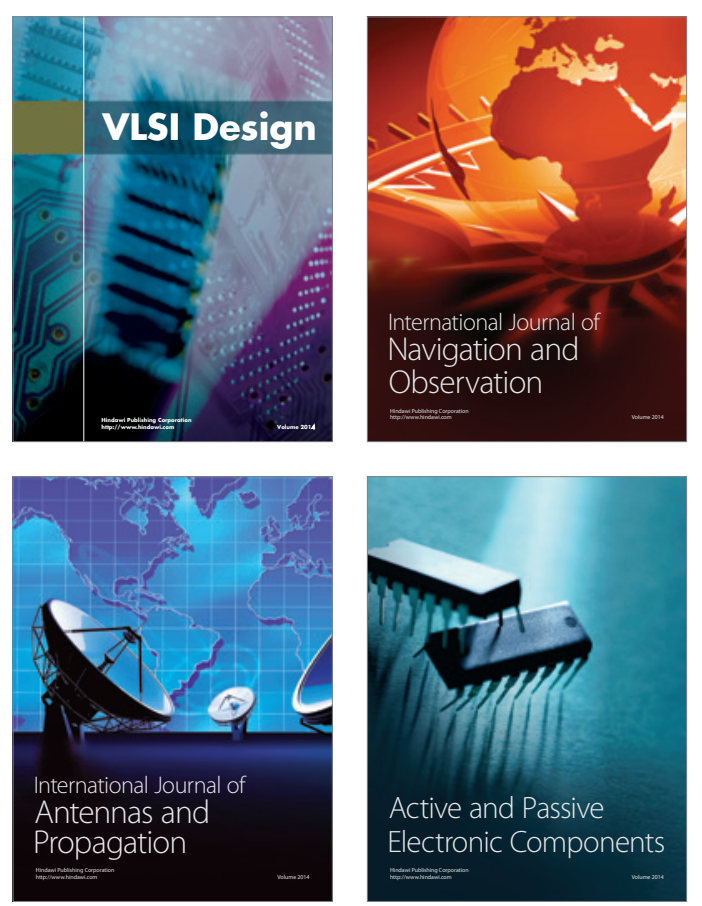
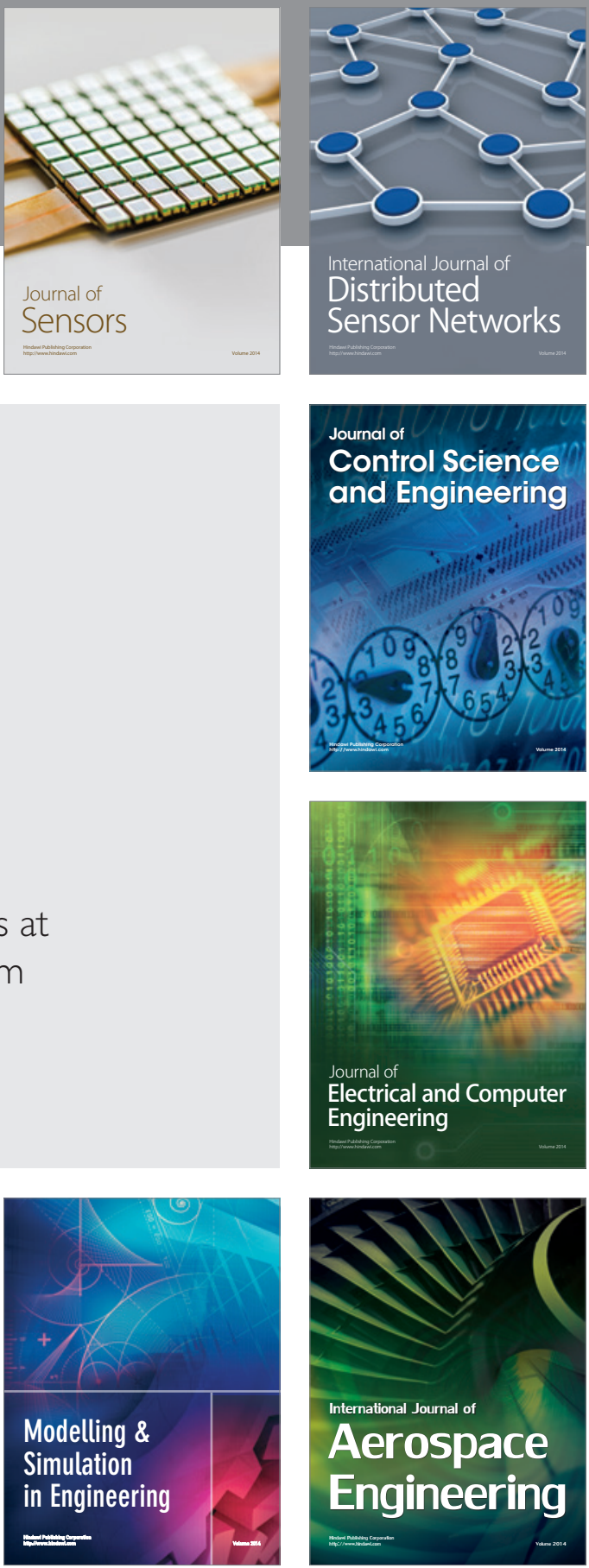

Journal of

Control Science

and Engineering
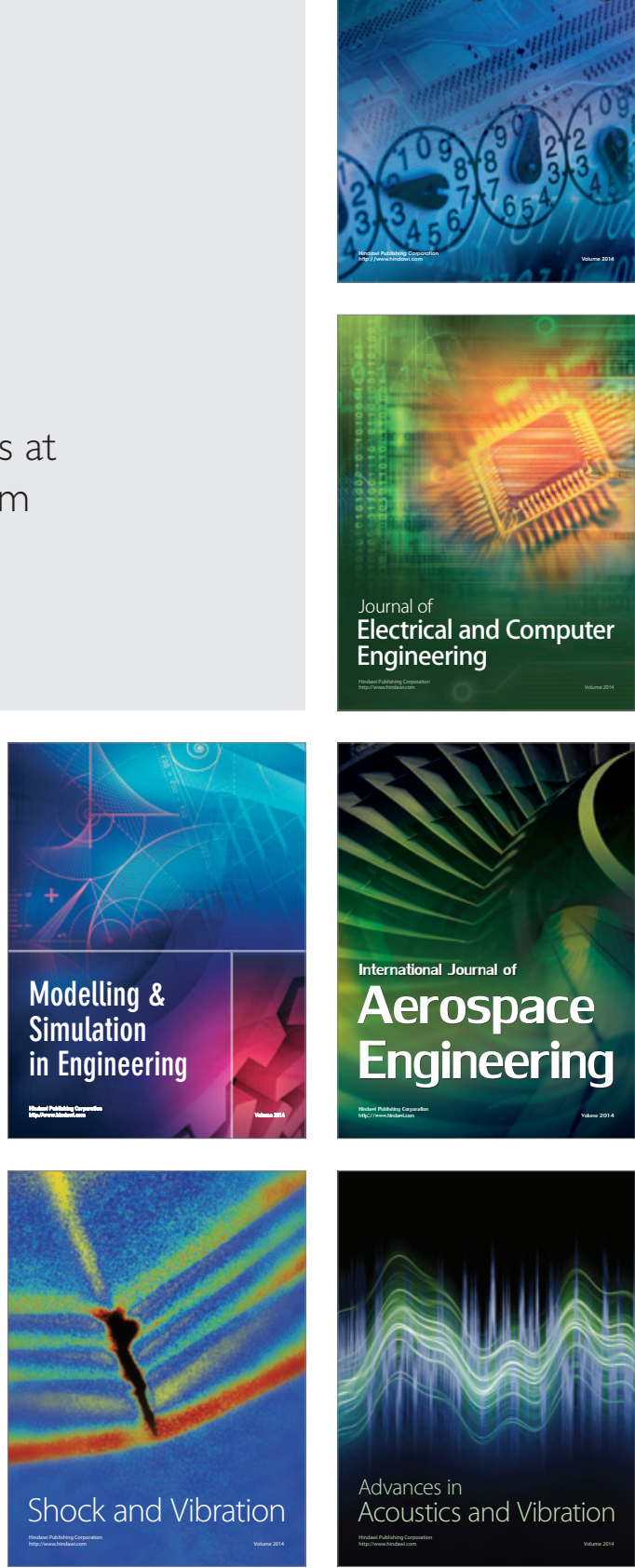